\title{
Energy-Saving D2D Wireless Networking Based on ACO and AIA Fusion Algorithm
}

\author{
Jiatong Li, ${ }^{1,2}$ Zhibo Li, ${ }^{1,2}$ Xuanying Li, ${ }^{1,3}$ and Cheng Wang $\mathbb{D}^{1,4}$ \\ ${ }^{1}$ Tianjin Key Laboratory of Wireless Mobile Communications and Power Transmission, Tianjin Normal University, \\ Tianjin 300387, China \\ ${ }^{2}$ Department of Communication Engineering, College of Electronic and Communication Engineering, Tianjin Normal University, \\ Tianjin 300387, China \\ ${ }^{3}$ Department of Electronic Information Science and Technology, College of Electronic and Communication Engineering, \\ Tianjin Normal University, Tianjin 300387, China \\ ${ }^{4}$ Department of Intelligence Science and Technology, College of Artificial Intelligence, Tianjin Normal University, \\ Tianjin 300387, China
}

Correspondence should be addressed to Cheng Wang; cwang@tjnu.edu.cn

Received 26 July 2021; Accepted 27 August 2021; Published 7 September 2021

Academic Editor: Xin Liu

Copyright (c) 2021 Jiatong Li et al. This is an open access article distributed under the Creative Commons Attribution License, which permits unrestricted use, distribution, and reproduction in any medium, provided the original work is properly cited.

Lower energy consumption and higher data rate have been becoming the key factors of modern wireless mobile communication for the improvement of user experiences. At present, the commercialization of $5 \mathrm{G}$ communications is gradually promoting the development of Internet of things (IoT) techniques. Due to the limited coverage capability of direct wireless communications, the indirect device-to-device (D2D) communications using information relay, in addition to the single 5G base station deployment, have been introduced. Along with the increase of information nodes, the relay devices have to undertake the nonnegligible extra data traffic. In order to adjust and optimize the information routing in D2D services, we present an algorithmic investigation referring to the ant colony optimization (ACO) algorithm and the artificial immune algorithm (AIA). By analyzing the characteristics of these algorithms, we propose a combined algorithm that enables the improved the iterative convergence speed and the calculation robustness of routing path determination. Meanwhile, the D2D optimization pursuing energy saving is numerically demonstrated to be improved than the original algorithms. Based on the simulation results under a typical architecture of $5 \mathrm{G}$ cellular network including various information nodes (devices), we show that the algorithmic optimization of $\mathrm{D} 2 \mathrm{D}$ routing is potentially valid for the realization of primitive wireless IoT networks.

\section{Introduction}

Since the late eighties of the last century, the Internet has been fast developing along with the progress of personal computers. Meanwhile, a concept of "connecting ubiquitous devices with Internet" emerged $[1,2]$ and further guided the research of the Internet of Things (IoT) for more comprehensive information sharing [3]. To date, the development of hardware techniques has made great progress. For instance, the fifth generation (5G) broadband communications employing high-frequency wavebands have unprecedentedly enhanced the data traffic performances [4]. However, it also has been noted that higher frequency leads to greater energy consumption due to the path loss of radio wave propagation [5]. As a result, the increasing dataflow requiring higher frequency inevitably encounters a power supply problem, which may lead to the unacceptable deployment density requirement of base stations $[6,7]$. Therefore, for the wireless networking of IoTs, the dataflow pressure of central base station is a critical challenge $[8,9]$. Under the current hardware development level, the direct routing scenarios using the traditional station-device communication scheme may not be suitable.

Focusing on the issues above, we consider that the networking of wireless IoTs may have to fully utilize the current hardware and pursuing energy-efficient scenarios 
from the algorithm aspect [10]. Following this idea, the algorithmic solutions improving the dataflow without additional energy consumption are commonly related to an indirect device-to-device (D2D) communication architecture, by which the information nodes (e.g., mobile devices) also undertake the message relay missions to reduce the data pressure of the central base station via proper routing solutions [11-13]. Thereby, figuring out an appropriate routing solution via programmed calculations and algorithms is significantly important to the D2D networking [14]. We regard that the $\mathrm{D} 2 \mathrm{D}$ routing is somehow similar to a classical mathematic problem which is the location of logistics distribution. To efficiently find out an available routing solution (may not be exactly optimized) under the specific constraint (e.g., energy efficiency in this work), the most well-known algorithms are the ant colony optimization (ACO) and the artificial immune algorithm (AIA) [15, 16]. First, ACO algorithms are usually adopted in wireless networking, which can be divided into two categories, the direct communication and the connectionless networks. In a direct one, all the message packets in the same loop propagate via a common path, which is selected by the preliminary setup state. On the contrary, the connectionless packets in the same loop propagate via different paths $[17,18]$. For the routing solution problem, the ACO algorithms envision the even and random manners of the information nodes and update the potentially optimal paths step-by-step [19]. However, these algorithms are still hindered by some limitations to this day, such as the blind search and local optimum problems. To overcome these issues, the routing scenarios employing AIA have been investigated for the fast convergence and the global optimization [20-22]. Although the information feedback in AIA programming is usually inefficient, which may lead to severely redundant iterations, the global optimizing advantage complementary to that of ACO algorithms may potentially open up a novel feasible algorithm appropriate to the iterative calculations for the wireless D2D routing paths [23].

Following these ideas, we proposed and a new algorithm, which combines the ACO and AIA principles, and quantitatively discussed the iterative calculation performances via numerical simulations. In particular, the physical environment of the D2D networking was designed based on the existing commercial 5G architecture, and the optimization constraint was accordingly selected as the reduce of energy consumptions $[10,24,25]$. According to the numerical simulation results, we showed that the newly designed algorithm significantly improves the D2D routing solutions, and the energy-saving performance may potentially enable the primitive wireless IoTs for sensor network applications.

\section{Algorithm}

2.1. ACO Principle. The ACO algorithm was originally developed for the vehicle routing problems (VRPs). Under this configuration, the feasible routing solutions are regarded as the paths of ants with similar mathematical models [18]. The paths of the entire ant population constitute a solution space. Here, we introduce the ACO algorithm into the $\mathrm{D} 2 \mathrm{D}$ routing model. After each iteration, the ant colony in ACO judges the optimization feasibility of each subset in the solution space, according to the newest pheromone concentration, and finds the optimal path. To maintain the universality, we set the number of ants $m(m \in[1, M])$, the number of nodes $n(n \in[1, N])$, and the distance between any two nodes $i$ and $j$ as $d_{i, j}(i, j=1,2, \ldots n)$. At the moment $t$, the pheromone concentration on a path connecting the nodes $i$ and $j$ is $\tau_{i, j}(t)$. In particular, at the initial time, $\tau_{i, j}(0)=\tau_{0}$. Considering that the next visiting node selected by ant $k$ is determined by the pheromone concentration on the connection path between corresponding relay nodes $s$, the propagation probability denoted $P_{i, j}^{k}(t)$ can be expressed as

$$
P_{i, j}^{k}(t)= \begin{cases}\frac{\left[\tau_{i, j}(t)\right]^{\alpha}\left[\eta_{i, j}(t)\right]^{\beta}}{\sum_{s \in B_{m}}\left\{\left[\tau_{i, j}(t)\right]^{\alpha}\left[\eta_{i, j}(t)\right]^{\beta}\right\}}, & \text { node } s \in B_{m}, \\ 0, & \text { otherwise, }\end{cases}
$$

where $\eta_{i, j}(t)$ is the heuristic function, representing the expected degree of the ant transfer from node $i$ to node $j$, and $B_{m}$ represents the set of relay nodes visited by ant $m$. Especially, $\alpha$ is the pheromone factor, greater $\alpha$ leads to higher pheromone concentration effect in transfer. Similarly, $\beta$ is the heuristic function factor; larger $\beta$ suggests higher probability of a relay node being selected. Regarding the modelling of ACO in path routing, we assume that there are $m$ ants in a quality of service (QoS) routing network, and the global and local pheromone updating rules are adopted [26]. Only the solution belongs to the global optimization path may lead to the increase of pheromone. We then select a constant $q_{0} \in[0,1]$ as the transfer factor and define a random variable $q \in[0,1]$ to describe the determination of the next node. While an ant $i$ in node $r$ selects the next node $s$ according to the following rules, if $q \leq q_{0}$, the next node maximizes $\left[\tau_{i, j}(t)\right]^{\alpha}\left[\eta_{i, j}(t)\right]^{\beta}$. In contrast, if $q>q_{0}$, the state transfer probability determined by equation (1) remains unchanged:

$$
\rho_{i}(r, s)= \begin{cases}\max [\operatorname{pheromone}(r, s)], & \text { node } s \in B_{m} \\ 0, & \text { otherwise. }\end{cases}
$$

According to equation (2), the ant transfer state determines the output probability results, which ensure the path optimization by searching the local maxima.

Following this principle, the ACO algorithms adopt the feedback mechanism and leave more concentrated pheromone in the paths, which indicates the better solutions. The positive feedback makes search processes continuously converging and finally approaching the optima. Any pheromone changes alter the surrounding environment and result in timely updates of the constraint result. Besides, if a suboptimal solution is utilized in the initial of calculation, the iterative convergence could be significantly accelerated. However, the rapid converging advantage may also induce a potential local optimum issue. As a result, other algorithms for the speed-optimum trade-off are necessary. 
2.2. AIA Principle. To get rid of the local optimum hindrance, the node prediction step planning for the more effective global routing suggests the AIA principle [27]. Under the classical AIA configuration, the antigens represent the subsets of a solution space, and the antibodies represent the coding sets of corresponding relay nodes. The antigen-antibody affinity indicates the recognition strength of a target node. Thus, the affinity function could be designed as a location model of the transmission of the node:

$$
\begin{aligned}
A_{v} & =\frac{1}{F_{v}} \\
& =\frac{1}{\sum_{i \in N}\left[\sum_{j \in M_{i}}\left(\omega_{i} d_{i, j} Z_{i, j}\right)\right]-C \sum_{i \in N} \min \left\{\left(\sum_{j \in M_{i}} Z_{i, j}\right)-1,0\right\}},
\end{aligned}
$$

where $F_{v}$ is the objective function, $N$ is the set of all nodes, $M_{i}$ is a subset including the nodes $i$ away from the base station closer than $s, w_{i}$ is the data volume of node $i, d_{i, j}$ is the distance between the nodes $i$ and $j, C$ is a manually selected large positive constant, and $Z_{i, j}$ is the distribution relation of the transmission data traffic between the central base station and a relay node. In the denominator of equation (3), the second term represents the punishment for the spatial solution violating the distance constraint. In addition, the similarity among antibodies is expressed as

$$
S_{v, s}=\frac{k_{v, s}}{L}
$$

where $k_{v, s}$ is the number of same bits of two antibodies $v$ and $s$ and $L$ is the length (number of bits) of antibody. Then, the antibody concentration can be obtained as

$$
C_{v}=\frac{1}{N} \sum_{j \in N} S_{v, s}
$$

Thereby, according to the affinity $A_{v}$ and the concentration $C_{v}$ above, the reproductive probability of an individual node is determined as

$$
P=\alpha \frac{A_{v}}{\sum A_{v}}+(1-\alpha) \frac{C_{v}}{\sum C_{v}},
$$

where $\alpha$ is an arbitrary constant factor. In equation (6), the individual fitness applies a positive effect on the expected reproduction probability. Higher affinity is helpful to the global optimization. However, due to the application of random searching, the efficiency of programming iteration and convergence are usually limited. On the basis of the discussion above, we considered to design a combined algorithm, which manages to concurrently take the advantages of ACO and AIA.
2.3. ACO/AIA Algorithmic D2D Routing. Under the current cellular network architecture, the deployment density increase of wireless devices (e.g., cellphones, unmanned equipment, and sensor array) results in the much greater data traffic pressure of the central base station. Thus, the traditional direct connection strategies are usually not available, especially on the energy consumption management aspect $[28,29]$. In order to handle this difficulty, the D2D networking exploiting wireless devices as the relay nodes has to be proposed as a potentially applicable approach. Because of the requirement of routing determination in D2D networking, the algorithm performances in converging speed and accuracy are significantly important. As a result, the selection of information propagation paths has to be readily determined with the global optimization. As we discussed above, two representative algorithms (i.e., ACO and AIA) support the iterative programming for the optimal routing determination, and occupy advantages complementary to each other [30]. Therefore, we tried to combine them together to concurrently preserve their advantages. In this work, the D2D routing is discussed as a typical wireless IoT networking mission under the existing 5G architecture [25].

The main idea of ACO/AIA fusion algorithm is replacing the worst antibody with the code represented by the best ant, thus to realize the information exchange between ACO and AIA. By using AIA, a series of starting times from the solution space can be selected to generate antibodies, and the ACO algorithm uses the solution space as the search range. On the basis of equation (1), we can further deduce the iterative update of pheromone $\tau_{i, j}(t)$ described by

$$
\tau_{i, j}(t+1)=(1-\psi) \tau_{i, j}(t)+\psi_{\tau_{0}},
$$

where $\psi \in(1,0]$ is a constant, $\tau_{0}=\left(m F_{n n}\right)^{-1}$ is the initial value of the pheromone trajectory between the channel nodes, and $F_{n n}$ is the fit value calculated by the nearest distance heuristic algorithm. Then, according to the guardian behavior in AIA, the optimal solution generated by ACO is used to stimulate the local search process. Meanwhile, the updating rules of pheromone trajectory are defined by

$$
\tau_{i, j}(t+1)=(1-\rho) \tau_{i, j}(t)+\rho \Delta \tau_{i, j}(t), \quad \rho \in(0,1],
$$

where $\rho$ is the volatile coefficient of pheromone and $\tau_{i, j}(t)$ is the pheromone increment of at $t$. At the time $t$, the ants pass through the node once, the local and global pheromones need updates. The pheromone parameters are adjusted according to equations (1) and (2) to prevent the local optimal solution. The affinity function $A_{v}$ obtained by equation (3) is used to define the final D2D algorithm pheromone update:

$$
\text { pheromone }(i, j)= \begin{cases}(1-p) \text { pheromone }(i, j)+\rho A_{v}, & \text { nodes } i, j \text { on path } \\ (1-p) \text { pheromone }(i, j)+b \rho A_{v}, & \text { otherwise. }\end{cases}
$$


In equation (9), $b \in(0,1)$ is a constant, which prevents the exceeding pheromone concentrations on multipath. Before the D2D routing optimization algorithm is complete, it maintains the iteration until the final convergence is obtained.

While the ACO/AIA fusion algorithm is proposed, we continue to discuss the constraint for the D2D networking optimization. Since our target is realizing a technically practical IoT scenario based on D2D routing, the optimization constraint is correspondingly set to make the total energy consumption minimized, at least lower than the traditional station-device direct communications. As the current communication techniques have almost approached the information channel capacity close to the Shannon limit, the data rate in this work is estimated by the upper limit. Then, based on the existing 5G architecture, we define the service zone covered by a single base station. Simply, we set a regular hexagon with the side length of $d_{\max }=60 \mathrm{~m}$. According to the Friis transmission formula, the data rate becomes a univariate function of the distance $d$. Therefore, the data rates of station-device $\left(R_{b s}\right)$ and device-device $\left(R_{d v}\right)$ are expressed as follows:

$$
\begin{cases}R_{b s}=W_{E H} \log _{2}\left[1+\frac{P_{b s_{\max }}^{\operatorname{tra}} G\left(c / 4 \pi f_{E H} d\right)^{2}}{p_{n} W_{E H}}\right], & d \in\left[0, d_{\max }\right], \\ R_{d v}=W_{S H} \log _{2}\left[1+\frac{P_{d v_{\max }}^{\operatorname{tran}} G\left(c / 4 \pi f_{S H} d\right)^{2}}{p_{n} W_{S H}}\right], & d \in\left[0, d_{\max }\right] .\end{cases}
$$

In equation (10), $f_{E H}=50 \mathrm{GHz}$ and $f_{S H}=2.4 \mathrm{GHz}$ indicate the carrier wave frequencies of base station (extreme high frequency, EHF) and wireless devices (special high frequency, SHF), respectively. Accordingly, $W_{E H}=500$ $\mathrm{MHz}$ and $W_{S H}=24 \mathrm{MHz}$ are the typical bandwidths of EHF and SHF, $P_{b s_{\max }}^{\text {tran }}=400 \mathrm{~W}$ and $P_{d v_{\max }}^{\text {tran }}=1 \mathrm{~W}$ are the typical power levels of EHF and SHF, $P_{n}=3.981 \times 10^{-21} \mathrm{WHz}^{-1}$ is the noise background power density, $c=3 \times 10^{8} \mathrm{~ms}^{-1}$ is the radiowave propagation speed, and $d \leq d_{\max }=60 \mathrm{~m}$ is the distance between any two nodes. The distribution of all the wireless devices, as well as the relay nodes, within a service zone is assumed to obey the Gaussian distribution. Therefore, in a representative loop $l$ containing $N_{l}$ nodes, the time length of $\mathrm{D} 2 \mathrm{D}$ communication $T_{l}$ is deduced as

$$
\begin{aligned}
T_{l} & =\sum_{n_{l}=1}^{N_{l}} T_{l, n_{l}} \\
& =\sum_{n_{l}=1}^{N_{l}}\left(\frac{1}{R_{l, n_{l}}} \sum_{i=1}^{n_{l}} I_{l, i}\right) .
\end{aligned}
$$

Based on equation (11), the total energy consumption can be calculated using $T_{l}$ values (including the values of devices $T_{l, n_{1}}$ and base station $\left.T_{l_{-} D 2 D}\right)$. Therefore, the optimization constraint for energy saving is expressed as

$$
E=P_{d v_{\max }}^{\operatorname{tran}} \sum_{n_{l}}^{N_{l}} T_{l, n_{l}}+P_{b s_{\max }}^{\operatorname{tran}} T_{l D 2 D^{\circ}}
$$

By using equation (12), the ACO/AIA algorithm can be executed as an iterative program, until the convergence indicates the minimal energy consumption.

\section{Simulation Results and Discussion}

On the basis of the algorithm and constraint we discussed above, the D2D routing for the networking of an energysaving IoT was testified via the programming iteration. After each iteration, the constraint result is calculated and fed back to the next iteration for the asymptotic minimization of constraint [30]. In particular, according to the existing 5G standard, the D2D sequence information amount sent by a base station is set as a constant of $10 \mathrm{Mb}$, and the data amount of a device is randomly generated obeying the Gaussian distribution within the range of 50-200 Mb. The programming scheme of out ACO/AIA fusion algorithm is illustrated in Figure 1, and the parameters used in the programming are provided in Table 1. In addition, to verify the performance advantage of our new algorithm, the classical ACO and AIA algorithms are also testified under the completely same conditions for comparisons [31].

Following the scheme in Figure 1, we first generate the information nodes (wireless devices) within a hexagonal cellular network service zone. As shown in Figure 2, the node positions including data amounts are randomly generated (30 nodes as a representative example in Figure 2(a)). The positions and data amounts of 30 nodes are also detailed in Table 2. While all the nodes are covered by a central base station, a traditional station-device direct connection model is constructed (Figure 2(b)).

We then executed the programming iterations based on the 30-node example (Figure 2) and presented the routing results in Figure 3. Comparing with the classical ACO and AIA algorithms, our novel fusion algorithm significantly reduces the large-span connections. And, the interferences between loops are also reduced. These numerical simulation results imply that the ACO/AIA fusion algorithm might be valid for the avoidance of local optimization disadvantages in the conventional algorithms.

Furthermore, the iterative optimization performances were quantitatively testified in the networking of 30-node situation. As shown in Table 3, all the results acquired from three algorithms approach stability after around 300-time iterations. However, the ACO/AIA algorithm exhibits much higher energy efficiency than the other two algorithms, as shown in Figure 4 . The much higher energy consumption levels of ACO and AIA algorithms, especially the classical ACO algorithm, provide the relatively high final results, which imply the local optimization issue as we mentioned above.

Moreover, in order to further verify the universality of our algorithm in the energy-saving optimization, the cases including more devices (information nodes) were tested. 


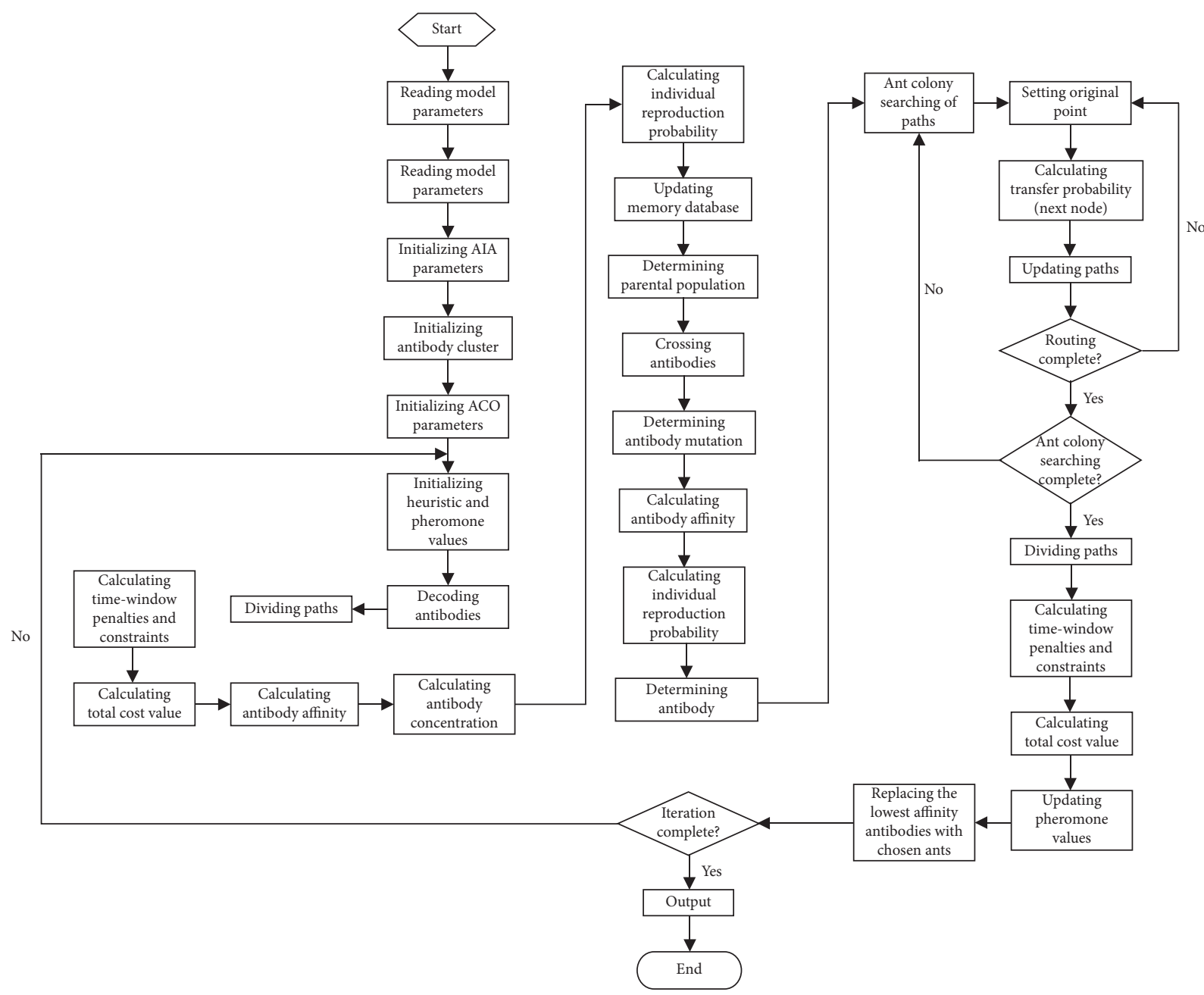

Figure 1: Programming scheme of the ACO/AIA algorithm for energy-saving optimization.

TABLE 1: Algorithm programming parameters.

Parameters

Value

Population size

Memory capacity

Crossover probability

Mutation probability

Diversity evaluation parameter

Pheromone index

Heuristic index

As shown in Figure 5, the representative examples of D2D networking of $70,150,200$, and 300 nodes optimized by using the ACO, AIA, and ACO/AIA fusion algorithms, respectively, are compared with each other. We discovered the consistent regularity that the ACO/AIA algorithm possesses the best energy efficiency and the convergence performance.
Besides, we would like to note that although D2D routing significantly enhances the energy efficiency, the time latency in indirect connections is an inevitable issue hindering the practical applicability. To overcome the timedelay hindrance, the final solution may still focus on the hardware developments, together with the algorithm investigations. 


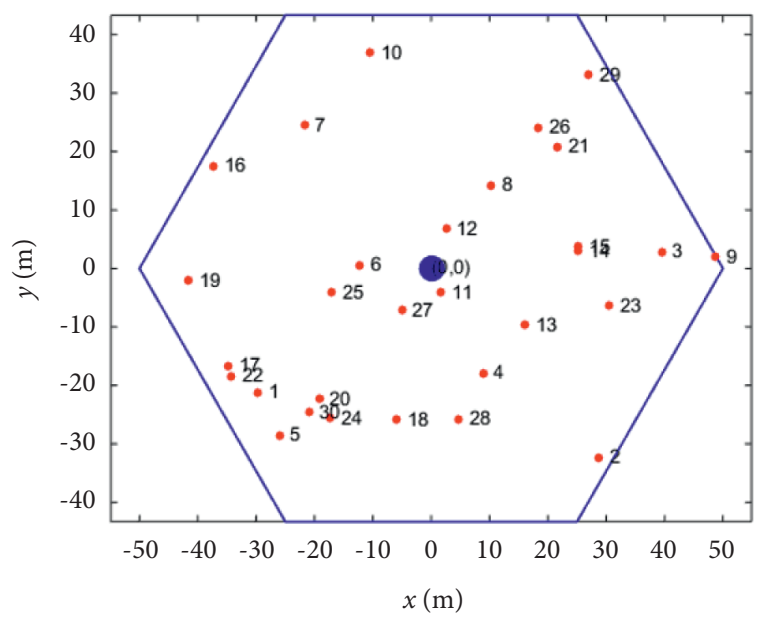

(a)

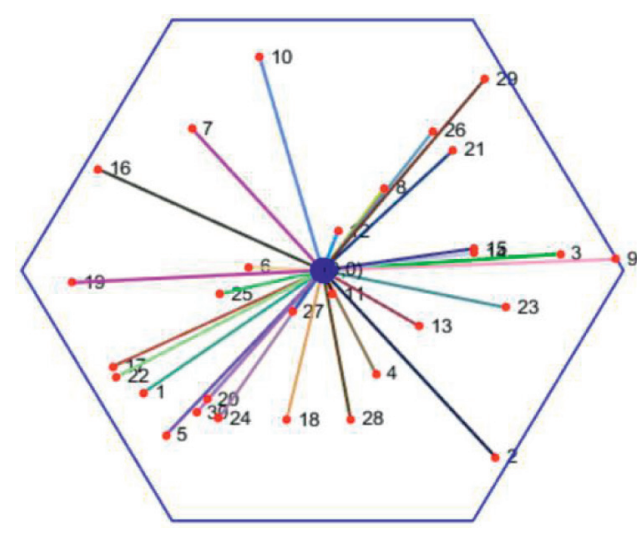

(b)

FIgURe 2: A representative wireless networking model containing 30 nodes. (a) Random generation of information nodes within a service zone. (b) Traditional direct communication strategy.

TABle 2: Node position and data amount information corresponding to Figure 2.

\begin{tabular}{|c|c|c|c|}
\hline Node & $x$-coordinate $(\mathrm{m})$ & $y$-coordinate $(\mathrm{m})$ & Data size $(\mathrm{Mb})$ \\
\hline 0 & 0 & 0 & 0 \\
\hline 1 & -29.75076725 & -21.1451 & 98 \\
\hline 2 & 28.8074986 & -32.3711 & 82 \\
\hline 3 & 39.51346795 & 2.855766 & 93 \\
\hline 4 & 8.864290527 & -17.8691 & 111 \\
\hline 5 & -26.04441642 & -28.5067 & 119 \\
\hline 6 & -12.29841177 & 0.593461 & 89 \\
\hline 7 & -21.67998262 & 24.53206 & 98 \\
\hline 8 & 10.27037863 & 14.25358 & 86 \\
\hline 9 & 48.77741011 & 1.931686 & 110 \\
\hline 10 & -10.43393433 & 36.8657 & 126 \\
\hline 11 & 1.760926681 & -4.10528 & 80 \\
\hline 12 & 2.552906914 & 6.706503 & 52 \\
\hline 13 & 16.18910379 & -9.73259 & 79 \\
\hline 14 & 25.22775935 & 2.95136 & 70 \\
\hline 15 & 25.23375902 & 3.787866 & 104 \\
\hline 16 & -37.32864055 & 17.53824 & 120 \\
\hline 17 & -34.73651287 & -16.5719 & 83 \\
\hline 18 & -5.951438079 & -25.8198 & 86 \\
\hline 19 & -41.53044014 & -2.10741 & 83 \\
\hline 20 & -19.18381344 & -22.3525 & 82 \\
\hline 21 & 21.70052876 & 20.78692 & 89 \\
\hline 22 & -34.21303177 & -18.425 & 58 \\
\hline 23 & 30.45716541 & -6.42097 & 112 \\
\hline 24 & -17.23243667 & -25.5631 & 76 \\
\hline 25 & -16.96341148 & -4.05674 & 61 \\
\hline 26 & 18.38068225 & 24.12656 & 101 \\
\hline 27 & -4.98049597 & -7.19577 & 143 \\
\hline 28 & 4.651289738 & -25.7734 & 108 \\
\hline 29 & 26.93717204 & 33.05046 & 102 \\
\hline 30 & -20.95107025 & -24.6377 & 59 \\
\hline
\end{tabular}



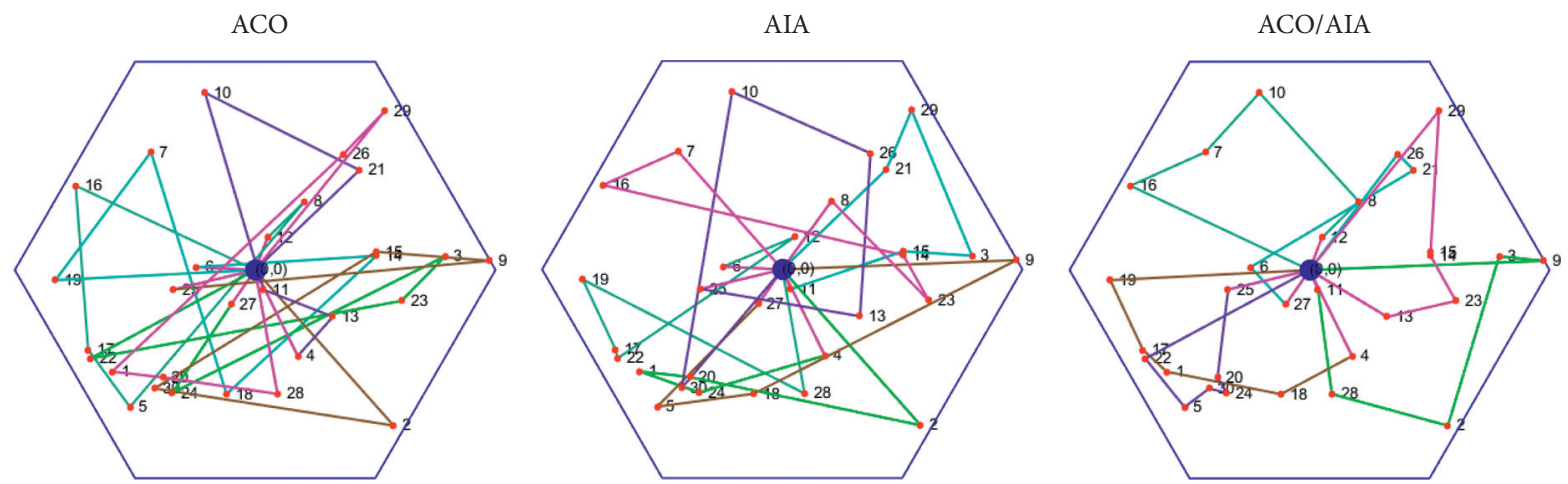

FIgURE 3: D2D routing results acquired from ACO, AIA, and ACO/AIA fusion algorithms (30 nodes in Figure 2).

TABle 3: Optimization trends of energy-saving D2D networking (30-node) along with the iterations of ACO, AIA, and ACO/AIA fusion algorithms.

\begin{tabular}{lccr}
\hline The number of iterations & \multicolumn{2}{c}{ Total energy consumption } & ACO/AIA \\
\hline 1 & ACO & 325210.4059 & 242842.1009 \\
2 & 292150.8678 & 325210.4059 & 242842.1009 \\
3 & 194461.987 & 325210.4059 & 240157.053 \\
$\ldots$ & 194461.987 & $\ldots$ & $\ldots$ \\
$\ldots$ & $\ldots$ & $\ldots$ & $\ldots$ \\
100 & $\ldots$ & 227245.5209 & $\ldots$ \\
$\ldots$ & 194461.987 & $\ldots$ & $\ldots$ \\
200 & $\ldots$ & 128434.1946 & 76446.35832 \\
$\ldots$ & 194461.987 & $\ldots$ & $\ldots$ \\
300 & $\ldots$ & 125626.1345 & 73241.97747 \\
301 & 194343.9423 & 125626.1345 & 73241.97746 \\
302 & 194343.9423 & 125626.1345 & 73241.97746 \\
303 & 194343.9423 & 125626.1345 & 73241.97746 \\
\hline
\end{tabular}

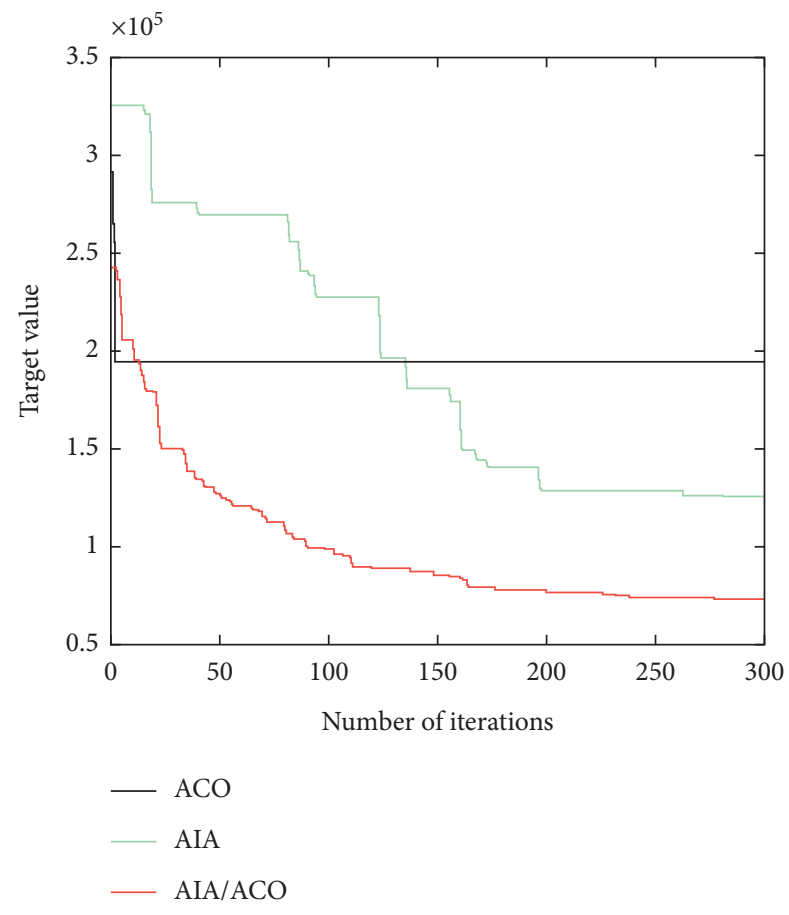

Figure 4: Energy efficiency optimization trends of ACO, AIA, and ACO/AIA fusion iterations. 

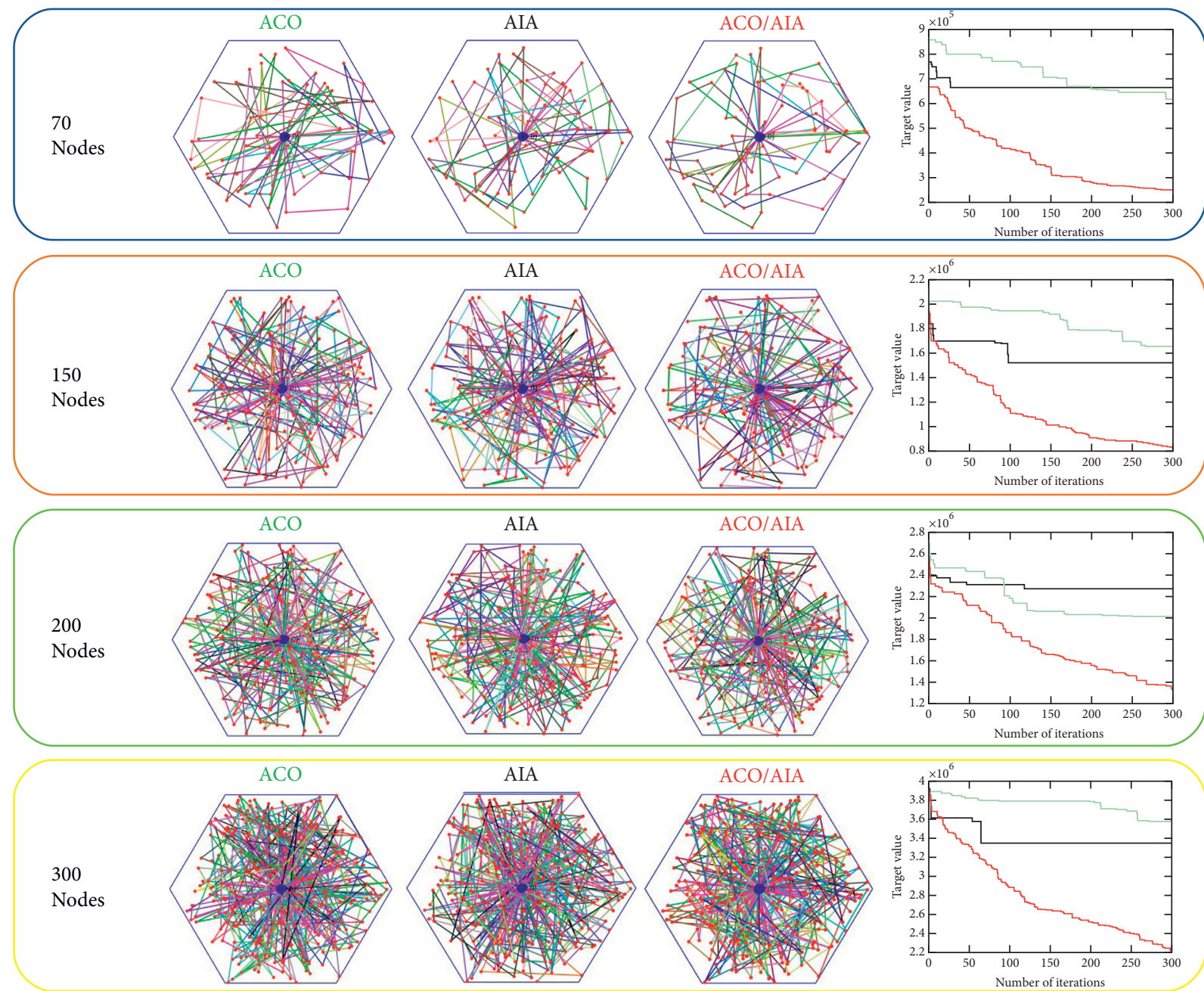

FIGURE 5: D2D routing results of 70, 150, 200, and 300 nodes optimized by ACO, AIA, and ACO/AIA fusion algorithms. Obviously, the ACO/AIA fusion algorithm provides much high iteration efficiency in the optimization for energy saving.

\section{Conclusion}

In summary, we investigated the $\mathrm{D} 2 \mathrm{D}$ routing scenarios for the energy-efficient networking from the algorithm aspect. Based on the numerical simulation results, we illustrated that the program iteration is a technical option for the construction of primitive D2D wireless IoTs, under the current hardware developing level. More importantly, we proposed a D2D routing algorithm based on the classical ACO and AIA algorithms. By testing the iterative optimization under the energy-saving constraint, the novel ACO/AIA fusion algorithm provides more satisfactory converging performances than traditional algorithms. The authors regard that the exploration of wireless IoT networking could be moved forward following this algorithmic method, concurrent to the hardware developments.

\section{Data Availability}

The data used to support the findings of this study are available from the corresponding author upon request.

\section{Conflicts of Interest}

The authors declare no conflicts of interest.

\section{Acknowledgments}

This work was partially funded by the National Natural Science Foundation of China (61901300 and 62101383).

\section{References}

[1] K. Ashton, "That 'internet of things' thing," RFID Journal, vol. 22, no. 7, pp. 97-114, 2009.

[2] B. Zhang, W. Liu, Q. Li et al., "Directional modulation design under a given symbol-independent magnitude constraint for secure IoT networks," IEEE Internet of Things Journal, 2020.

[3] L. Atzori, A. Iera, and G. Morabito, "The internet of things: a survey," Computer Networks, vol. 54, no. 15, pp. 2787-2805, 2010.

[4] W. Saad, M. Bennis, and M. Chen, "A vision of $6 \mathrm{G}$ wireless systems: applications, trends, technologies, and open research problems," IEEE Network, vol. 34, no. 3, pp. 134-142, 2019. 
[5] J. Wu, H. Shen, R. Yin, Y. He, J. Li, and C. Wang, "Displaying images on a plane of rotation via mutual inductance coupling wireless controls," Ad Hoc Networks, vol. 107, Article ID 102256, 2020.

[6] B. Zhang, W. Liu, J. Ma et al., "Sparse antenna array based positional modulation design with a low-complexity metasurface," IEEE Access, vol. 8, Article ID 177640, 2020.

[7] C. Wang, B. Zhang, Y. Li, and X. Zhao, "Suspended graphene hydroacoustic sensor for broadband underwater wireless communications," IEEE Wireless Communications, vol. 27, no. 5, pp. 44-52, 2020.

[8] B. Nishina and Q. Chen, "Estimation of equivalent current distribution of modulated EM radiation source," IEEE Transactions on Antennas and Propagation, vol. 64, no. 4, pp. 1334-1341, 2016.

[9] B. Zhang, W. Liu, Y. Li, X. Zhao, and C. Wang, "Directional modulation design under maximum and minimum magnitude constraints for weight coefficients," Ad Hoc Networks, vol. 98, Article ID 102034, 2020.

[10] W. Ejaz, M. Naeem, A. Shahid, A. Anpalagan, and M. Jo, "Efficient energy management for the internet of things in smart cities," IEEE Communications Magazine, vol. 55, no. 1, pp. 84-91, 2017.

[11] H. Wu, X. Gao, S. Xu, D. O. Wu, and P. Gong, "Proximate device discovery for D2D communication in LTE advanced: challenges and approaches," IEEE Wireless Communications, vol. 27, no. 4, pp. 140-147, 2020.

[12] B. Zhang, W. Liu, Y. Li, X. Zhao, C. Zhang, and C. Wang, "Symbol-independent weight magnitude design for antenna array based directional modulation," Ad Hoc Networks, vol. 101, Article ID 102097, 2020.

[13] K. Doppler, M. Rinne, C. Wijting, C. Ribeiro, and K. Hugl, "Device-to-device communication as an underlay to LTEadvanced networks," IEEE Communications Magazine, vol. 47, no. 12, pp. 42-49, 2009.

[14] C. Di, B. Zhang, Q. Liang, S. Li, and Y. Guo, "Learning automata-based access class barring scheme for massive random access in machine-to-machine communications," IEEE Internet Things Journal, vol. 6, no. 4, pp. 6007-6017, 2018.

[15] I. Aydin, M. Karakose, and E. Akin, "A multi-objective artificial immune algorithm for parameter optimization in support vector machine," Applied Soft Computing, vol. 11, no. 1, pp. 120-129, 2011.

[16] M. Dorigo and C. Blum, "Ant colony optimization theory: a survey," Theoretical Computer Science, vol. 344, no. 2-3, pp. 243-278, 2005.

[17] B. Zhang, W. Liu, Y. Li, X. Zhao, and C. Wang, "Directional modulation design under a constant magnitude constraint for weight coefficients," IEEE Access, vol. 7, Article ID 154711, 2019.

[18] D. Martens, M. D. Backer, R. Haesen, J. Vanthienen, M. Snoeck, and B. Baesens, "Classification with ant colony optimization," IEEE Transactions on Evolutionary Computation, vol. 11, no. 5, pp. 651-665, 2007.

[19] M. Dorigo, M. Birattari, and T. Stutzle, "Ant colony optimization," IEEE Computational Intelligence Magazine, vol. 1, no. 4, pp. 28-39, 2006.

[20] M. Pedemonte, S. Nesmachnow, and H. Cancela, "A survey on parallel ant colony optimization," Applied Soft Computing, vol. 11, no. 8, pp. 5181-5197, 2011.

[21] B. Zhang, W. Liu, Q. Li et al., "Metasurface based positional modulation design," IEEE Access, vol. 8, Article ID 113807, 2020 .
[22] D. Dasgupta, “Advances in artificial immune systems," IEEE Computational Intelligence Magazine, vol. 1, no. 4, pp. 40-49, 2006.

[23] S. Cui, A. J. Goldsmith, and A. Bahai, "Energy-efficiency of MIMO and cooperative MIMO techniques in sensor networks," IEEE Journal on Selected Areas in Communications, vol. 22, no. 6, pp. 1089-1098, 2004.

[24] M. Waqas, Y. Niu, Y. Li et al., "Mobility-aware device-todevice communications: principles, practice and challenges," IEEE Communications Surveys \& Tutorials, vol. 18, no. 7, pp. 3658-3668, 2019.

[25] K. David and H. Berndt, "6G vision and requirements: is there any need for beyond 5G?" IEEE Vehicular Technology Magazine, vol. 13, no. 3, pp. 72-80, 2018.

[26] S. Reddy, "Multi-path selection based on fractional cuckoo search algorithm for QoS aware routing in MANET," Sensor Review, vol. 39, no. 2, pp. 218-232, 2019.

[27] Q. Yu, J. Ren, J. Zhang et al., "An immunology-inspired network security architecture," IEEE Wireless Communication, vol. 27, no. 5, pp. 168-173, 2020.

[28] L. Zou, J. Chen, L. Lv, and B. He, "Capacity enhancement of D2D aided coordinated direct and relay transmission using NOMA," IEEE Communications Letters, vol. 24, no. 10, pp. 2128-2132, 2020.

[29] P. Popovski and H. Yomo, "Wireless network coding by amplify-and-forward for bi-directional traffic flows," IEEE Communications Letters, vol. 11, no. 1, pp. 16-18, 2007.

[30] J. Li, M. Li, J. He, W. Shi, and C. Wang, "D2D routing aided networking for efficient energy consumption management of wireless IoT," Ad Hoc Networks, vol. 123, Article ID 102636, 2021.

[31] R. Shafin, L. Liu, V. Chandrasekhar, H. Chen, J. Reed, and J. C. Zhang, "Artificial intelligence-enabled cellular networks: a critical path to beyond-5G and 6G," IEEE Wireless Communications, vol. 27, no. 2, pp. 212-217, 2020. 